\title{
Manipulation of Backscattering From a Dielectric Cylinder of Triangular Cross-Section Using the Interplay of GO-Like Ray Effects and Resonances
}

\author{
Ilya O. Sukharevsky, Member, IEEE, Alexander I. Nosich, Fellow, IEEE, and Ayhan Altintas, Senior Member, IEEE
}

\begin{abstract}
A triangular dielectric cylinder (dielectric prism) of the size, in cross-section, comparable to or moderately larger than the wavelength is a scatterer, which blends together two different types of electromagnetic behavior: geometrical optics (GO) and resonance. As shown in this paper, the first is responsible, for instance, for enhanced reflection from an isosceles $90^{\circ}$ prism, if illuminated from the base. The second is responsible for the peaks in the total scattering and absorption cross-sections (ACSs) at the natural-mode frequencies. The numerical analysis is performed by solving the well-conditioned Muller-type boundary integral equation (IE) discretized using an algorithm with controlled accuracy.
\end{abstract}

Index Terms-Dielectric prism, Muller integral equation (IE), natural resonances, radar cross-section (RCS), scattering.

\section{INTRODUCTION}

D ESPITE the anticipation that the triangular cross-section dielectric cylinders can be useful in applications due to their obvious geometrical optics (GO) stealth properties, the diffraction of waves by such a canonical scatterer seems to have escaped the attention of researchers. This is even more evident if one looks for the information on the radar cross-section (RCS) behavior in the wide range of frequencies and for varying parameters such as the apex angle, dielectric contrast, and the angle of incidence. Only partial studies of this scatterer or open resonator have been performed with asymptotic methods [1]-[3] valid for electrically huge scatterers.

The tools of the full-wave numerical analysis of medium-size dielectric scatterers are normally associated with the volume and boundary integral equations (IEs). The latter equations are

Manuscript received September 30, 2014; revised December 17, 2014; accepted February 09, 2015. Date of publication February 16, 2015; date of current version May 01, 2015. The work of I. O. Sukharevsky was supported by the Scientific and Technological Research Council of Turkey (TUBITAK) via 2221 Fellowship Program.

I. O. Sukharevsky is with the Communication and Spectrum Management Research Center (ISYAM), Bilkent University, 06800 Ankara, Turkey (e-mail: i.sukharevsky@gmail.com).

A. I. Nosich is with the Laboratory of Micro and Nano Optics of the Institute of Radio-Physics and Electronics of the National Academy of Sciences of Ukraine, Kharkiv 61085, Ukraine.

A. Altintas is with the Department of Electrical and Electronics Engineering, Bilkent University, 06800 Ankara, Turkey, and also with the Faculty of Engineering and Natural Sciences, Abdullah Gul University, 38039 Kayseri, Turkey.

Color versions of one or more of the figures in this paper are available online at http://ieeexplore.iee.org.

Digital Object Identifier 10.1109/TAP.2015.2404338 usually more attractive as demanding the discretization of only the scatterer contour instead of its area [4]-[22].

However, one should keep in mind that many forms of the boundary IEs are contaminated by the presence of spurious eigenvalues [15], [16]. For instance, all the works [3]-[14] dealt with such IE although this fact was fully acknowledged only in the paper [10]. The spurious eigenvalues are the real numbers that are the eigenvalues of the interior electro-magnetic problem where the boundary is assumed perfectly electrically conducting (PEC), and the inside filling is assigned material parameters of the outer medium (e.g., free space). The spurious eigenvalues have no physical meaning and spoil the computation of the frequency scans of RCS and other characteristics by producing a "forest" of false spikes.

Note that the analytical regularization of such defective IEs, i.e., their reduction to the Fredholm second-kind matrix equations, achieved, for instance, using their projection to specially tailored Galerkin basis functions [11], [14] does not remove the defect mentioned. Still it helps shrink the false spikes produced by spurious eigenvalues to narrow peaks whose width gets smaller for the larger discretizations.

As discussed in [17] and [18], the spurious eigenvalues are absent for the Muller boundary IE (MBIE) [19], which, in the two-dimensional (2-D) case, is a pair of coupled second-kind IEs for the field components tangential to the scatterer contour [16], [17], [19],. This is unlike the combined-field IE [18], which only pushes the spurious eigenvalues off from the real axis. As the kernels of MBIE are smooth or integrable functions [17], this is a Fredholm second-kind IE, and therefore the Fredholm theorems guarantee the convergence of conventional projection (moment-method) discretizations. In the past, MBIE has been used in [20] and [21] together with the collocation algorithm (i.e., meshing the contour and using the local basis functions) to demonstrate the potentialities of approach. In [22] and [23], MBIE has been alternatively projected to the trigonometric polynomials as global basis functions, to exploit their regularizing properties with respect to MBIE kernels.

It must be emphasized that the Nystrom-type meshless discretization, which exploits interpolation polynomials and quadrature formulas, also guarantees the convergence. Here, we understand the convergence in mathematical sense, as ability to minimize the error of computations, to machine precision, by using progressively larger orders of discretization. In the 2-D scattering by dielectric objects, several Nystrom-type schemes have been proposed, all of them for the contours allowing 
smooth $2 \pi$-periodic parameterization [17], [18]. In particular, the economic and robust algorithm of [17] exploits splitting all kernels of MBIE into logarithmic and smooth parts and uses two different quadrature formulas for replacing the integrals with finite sums. However, this treatment must be modified if the contour is only piece-wise smooth, which is considered in this paper.

In this paper, we use MBIE to study accurately the interplay of ray effects and resonances in a medium-sized triangular dielectric cylinder (prism) illuminated by a plane wave. Our numerical algorithm implies segmentation of contour to minimum number of regular parts and further uses a numerical algorithm explained and validated in [24].

The remaining part of this paper is organized as follows. In Section II, we formulate the scattering problem and briefly outline the derivation of MBIE and its numerical solution adapted for piece-wise smooth contours. In Section III, we present the numerical results related to the RCS analysis of several triangular dielectric cylinders. Section IV summarizes the conclusion.

The time dependence $\exp (-i \omega t)$ is implied and omitted.

\section{Problem Formulation, Method Description, AND MAIN CHARACTERISTICS}

We consider an electromagnetic plane wave incident at the angle $\beta$, counted from the $-x$ axis, on a uniform dielectric cylinder of triangular cross-section with a relative dielectric constant $\varepsilon$ (Fig. 1). To ensure that the cylinder contour $L$ satisfies the Lyapunov condition that is typical for boundary IEs [17]-[23], the apexes of the triangle are smoothened by the circular arcs of the radius $c$. As we have found numerically, making the rounding-arc radius smaller than $c<0.05 \lambda$ does not change the first four digits in the RCS value.

The $E_{z}$ or $H_{z}$ component of the field scattered by cylinder $u(x, y)$ must satisfy the following boundary-value problem: 2-D Helmholtz equation outside and inside $L$, the tangential components continuity at $L$, Sommerfeld condition at infinity, and the finiteness of the field power in any bounded domain. The solution of such problem is known to exist and be unique.

A brief outline of the MBIE method is as follows. The Green's formula enables one to express the fields inside and outside $L$ in terms of the limiting values of the fields and their normal derivatives on $L$. By applying the boundary conditions, a set of two Fredholm second-kind IEs is obtained, known as MBIE [17]-[22]. For its solution, we use an algorithm adapted to the piece-wise smooth contour-see [24]. Here, the logarithmic singularities are integrated analytically while the integrals of regular functions are approximated by quadratures.

The algorithm developed guarantees point-wise convergence of the numerical solution, i.e., the possibility of making the computational error smaller by solving progressively larger matrices. Note that the required accuracy can be achieved for an arbitrary set of parameters, i.e., for any value of the contrast between the cylinder and the host medium. It is free from the inaccuracies intrinsic to finite-difference timedomain (FDTD) approximations [25]-[27] near to the sharp natural-mode resonances, as well as near to the non-physical

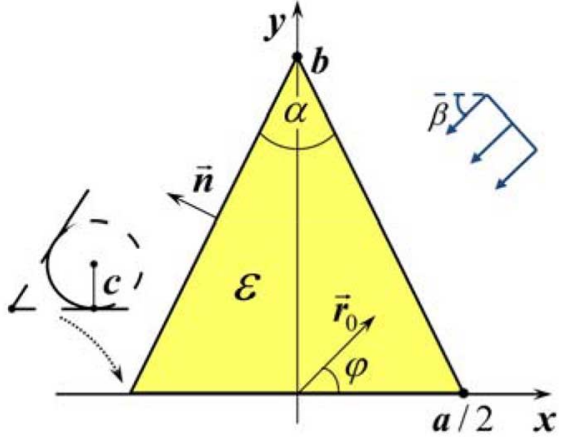

(a)

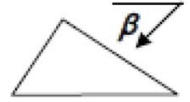

(b)
Fig. 1. (a) Cross-section of an isosceles triangular cylinder (prism) of the relative dielectric permittivity $\varepsilon$, the base $a$, and the height $b$, with rounded edges (see text for details), illuminated by a plane wave incident under the angle $\beta$; (b) Geometry of a Pythagorean-triangle prism excitation.

"numerical resonances" caused by the spurious eigenvalues occurring if using non-MBIEs [15], [16].

Considering the field scattered by the prism in the far zone $(r \rightarrow \infty)$, we use the asymptotic expressions for the Hankel functions and their derivatives, in the kernels of the integral representations, and arrive at the following expression: $u(r)=$ $\left(8 \pi k_{0} r\right)^{-1 / 2} e^{i\left(k_{0} r+\pi / 4\right)} \Phi(\beta)$. Then the monostatic RCS (the same as backward scattering cross-section) and the total scattering cross-section (TSCS) are obtained as, respectively,

$$
\begin{aligned}
\sigma_{b} & =\left(4 k_{0}\right)^{-1}|\Phi(\beta)|^{2} \\
\sigma_{s c} & =\left(8 \pi k_{0}\right)^{-1} \int_{0}^{2 \pi}|\Phi(\varphi)|^{2} d \varphi .
\end{aligned}
$$

Here, the far-field scattering pattern is

$$
\Phi(\varphi)=\int_{L} e^{-i k_{0}\left(\vec{r}_{0} \cdot \vec{x}\right)}\left[i k_{0}\left(\vec{n} \cdot \vec{r}_{0}\right) u(\vec{x})+p \frac{\partial u(\vec{x})}{\partial n}\right] d s
$$

$u$ and $\partial u / \partial n$ are the limiting values of the inner field and its normal derivative on $L, k_{0}$ is the free-space wavenumber, $\vec{r}_{0}=(\cos \varphi, \sin \varphi)$ is the unit radius-vector in the far zone, $\vec{x}$ is the radius-vector of a point on $L, \vec{n}$ is the outward normal unit vector, and $p=1$ in the E-polarization case or $1 / \varepsilon$ in the $\mathrm{H}$-polarization case.

Another value of interest is the absorption cross-section (ACS), which can be found from the Optical Theorem

$$
\sigma_{\mathrm{abs}}=-\operatorname{Im} \Phi(\beta+\pi) / k_{0}-\sigma_{s c} .
$$

Further we will analyze the behavior of RCS and use TSCS and ACS as auxiliary characteristics.

\section{NUMERICAL RESUlts: RAY EFFECTS}

\section{A. GO Effect of Stealth Scatterer at the Edge-On Incidence}

In Fig. 2, we present the dependences of RCS of five scatterers shaped as isosceles triangles with the apex angle values of $30^{\circ}, 60^{\circ}, 90^{\circ}, 120^{\circ}$, and $150^{\circ}$, on the angle of the planewave incidence $\beta$ in polar coordinates. The frequency is fixed as $k a=15$ (i.e., $a \approx 2.39 \lambda=4.78 \lambda \varepsilon^{-1 / 2}$ ) and the relative 


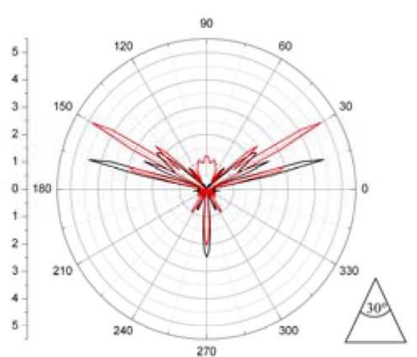

(a)

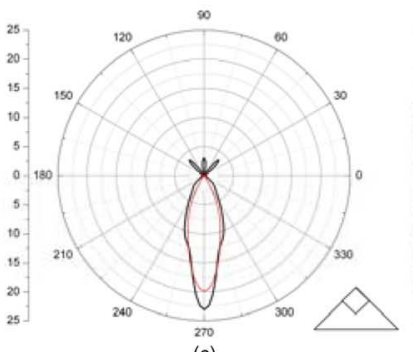

(c)

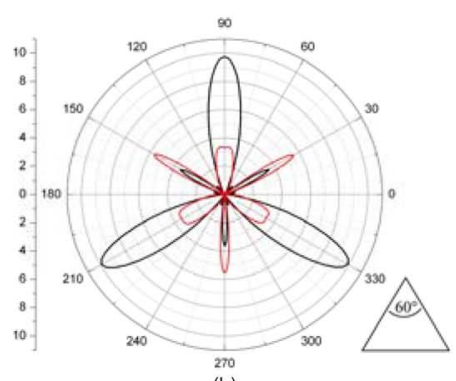

(b)

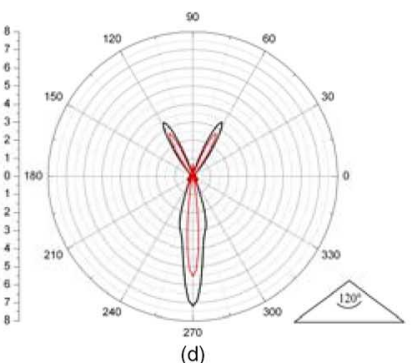

(d)

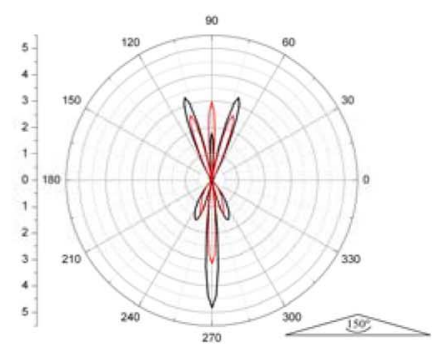

(e)

Fig. 2. Monostatic RCS of isosceles triangular cylinders with $\varepsilon=4$ and $k a=$ 15 as a function of the angle of incidence $\beta$ for the apex angle values (a) $\alpha=$ $30^{\circ}$; (b) $60^{\circ}$; (c) $90^{\circ}$; (d) $120^{\circ}$; and (e) $150^{\circ}$ in the case of the E- (black curves) and the H-polarization (red curves).

dielectric permittivity as $\varepsilon=4$. Two polarizations differ by the color of curves-see the figure captions.

If the plane wave is incident on one of the edges of a mediumsize triangular scatterer, one can expect a reduction of RCS because of the GO-like deflection of the wave by the adjacent flat facets. Our computations confirm this anticipation. At edgeon incidence, the RCS values are small—see panels (a) and (c) to (e) - unless the frequency is close to internal resonance as on panel (b) (see below on resonances).

\section{B. GO Effect of Enhanced Reflections From Flat Facets}

The effect of the extended flat facets on the RCS is well observable: if a facet is placed normal to the incident wave, RCS displays a sharp maximum.

This is true for any apex angle $\alpha$. For instance, if $\beta=$ $270^{\circ}$, i.e., the triangle base is under the normal incidence, such effect is visible on the panels (a), (c), (d), and (e). Other two facets contribute similarly at the angles of incidence $\beta=$ $90^{\circ} \pm \alpha / 2$, in full accordance with GO. Here, it is useful to remember that RCS of a PEC flat strip of the width $a$ has sharp maximum at the normal incidence and quickly decays if the angle of illumination $\theta$, measured from the normal, deviates from it: $\sigma_{b}=4 k a^{2}\left[1-O\left(\theta^{4}\right)\right]$ [28]. Although a flat

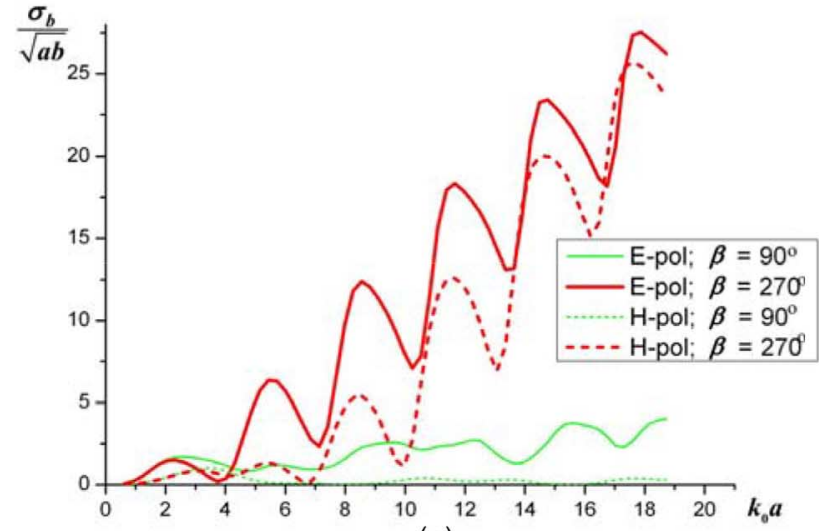

(a)

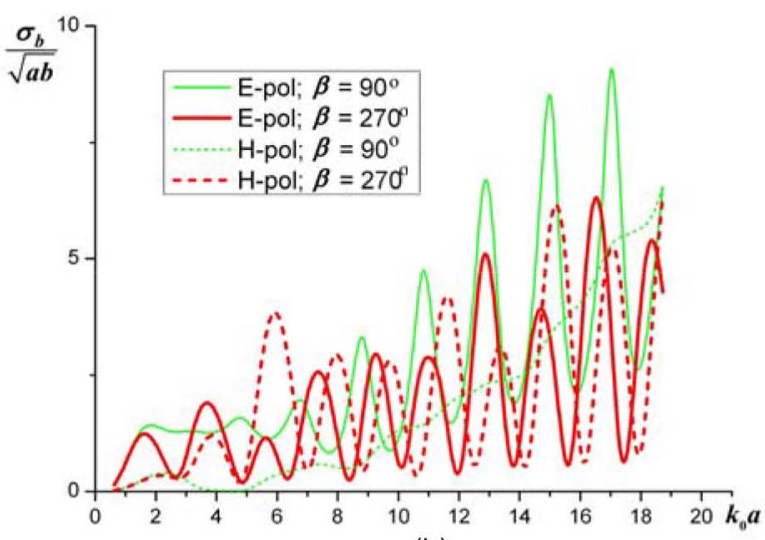

(b)

Fig. 3. Dependences of the normalized RCS on the normalized frequency for the E- and H-polarized plane wave incidence at triangular dieletric cylinders with $\varepsilon=4$ and the (a) apex angles $\alpha=90^{\circ}$ and (b) $60^{\circ}$.

facet of triangular dielectric cylinder is not a perfect mirror, it shows similar behavior of RCS as a function of the angle of incidence.

This observation is further supported by the plots of the RCS dependences on the normalized frequency $k_{o} a$ shown in Fig. 3 (see the curves for $\beta=270^{\circ}$ ). In the case of the facet-on incidence in either polarization (red curves), the normalized RCS displays the same linear growth with $k_{o} a$ as a PEC strip, however modulated with oscillations due to the internal resonances (see Section IV).

\section{GO Effect of Corner Reflector for $\alpha=90^{\circ}, \beta=270^{\circ}$}

A right-triangle isosceles prism $\left(\alpha=90^{\circ}\right)$ shows another interesting GO effect provided that the plane wave is incident on the base. Such a scatterer behaves similarly to a $90^{\circ}$ corner reflector [29] — see the RCS dependence on $\beta$ and a sketch in of [3, Fig. 10]. In the GO approximation, every ray impinging on the flat base experiences two refractions in accordance with Snell's law and two internal reflections and gets out of the prism in the backward direction. Note that the peak values of RCS at the normal base-on incidence are some five to ten times larger than for the apex angles $\alpha$ other than $90^{\circ}$ [compare to panels (a), (d), and (e)]. The same conclusion can be made from the plots of RCS given in Fig. 3. 


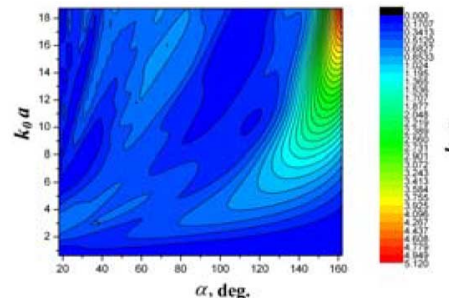

(a)

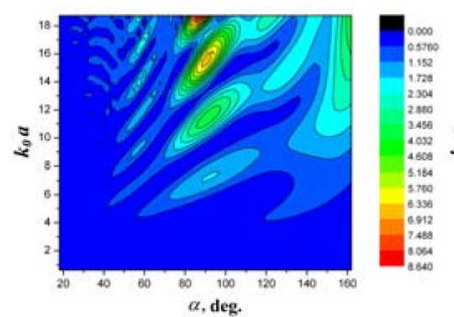

(c)

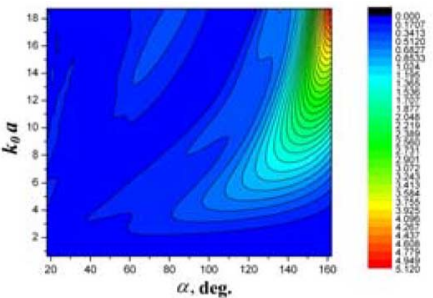

(b)

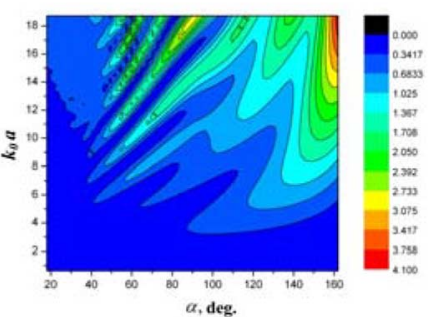

(d)
Fig. 4. Contour plots of the normalized RCS of triangular cylinder with $\varepsilon=2.5$ as a function of the apex angle $\alpha$ and parameter $k_{0} a$ in the (a) and (c) E-polariztion case and (b) and (d) H-polarization case. The incidence angle is $\beta=90^{\circ}$ in (a) and (b) and $\beta=270^{\circ}$ in (c) and (d).

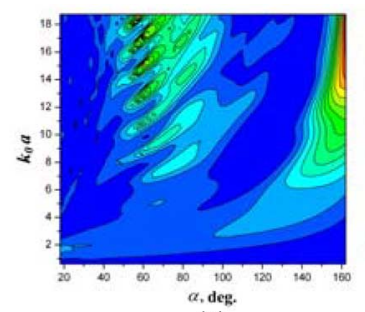

(a)

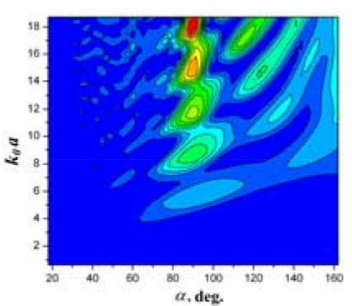

(c)
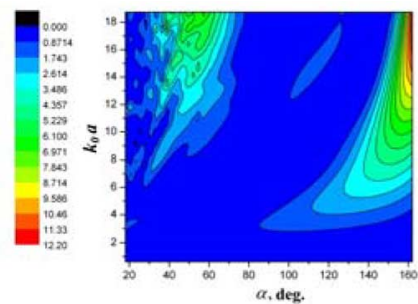

(b)
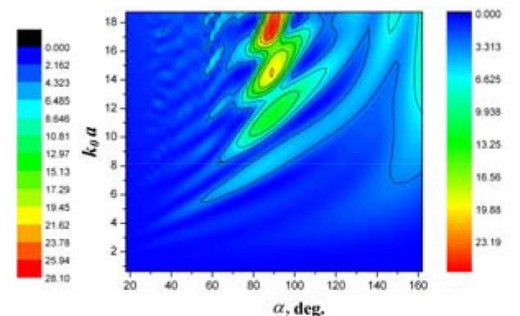

(d)
Fig. 5. Same as in Fig. 4, however, for $\varepsilon=4$.

The equilateral triangle RCS [see Fig. 2(b) and Fig. 3(b)] needs separate discussion (see Section IV-C); however, it also shows enhanced RCS if illuminated normally to one of the facets. Note that both the right-triangle isosceles and the equilateral triangular prism RCS dependences of the frequency are modulated with nearly periodic peaks, which betray the presence of resonances of scatterer.

\section{Enhanced RCS of Flattened Triangles, $\alpha \rightarrow 0^{\circ}$ or $180^{\circ}$}

In the limiting case of a "flattened" triangle where the apex angle $\alpha$ is close to $0^{\circ}$ or $180^{\circ}$, our dielectric scatterer behaves similarly to a thin flat dielectric strip. The properties of a flat dielectric strip have been presented, for instance, in [30]. Such a strip, if its width is at least a few wavelengths in free space, behaves as an imperfect flat mirror. It shows high levels of RCS

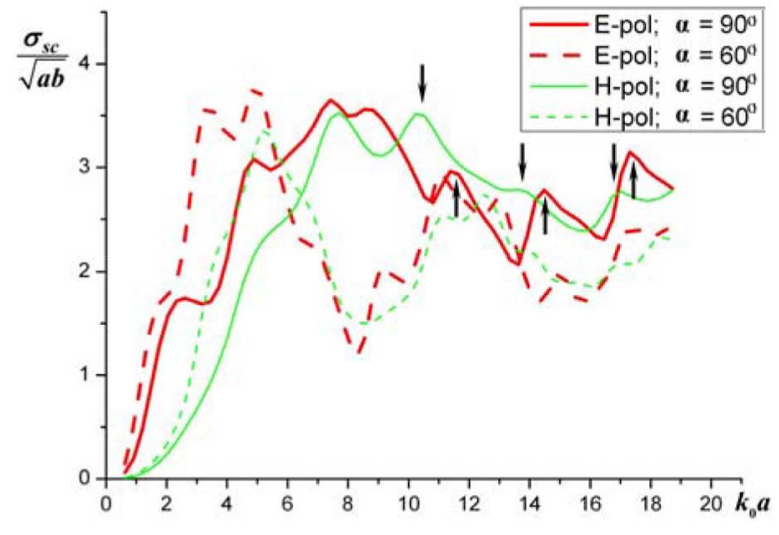

Fig. 6. Normalized TSCS of a triangular dielectric cylinder with $\varepsilon=4$ as a function of the normalized frequency $k_{0} a$ for two alternative polarizations at $\beta=90^{\circ}$ or $270^{\circ}$ and two values of the apex angle, $\alpha=60^{\circ}$ and $90^{\circ}$.

only under normal illumination; otherwise it deflects the wave according to quasi-optical stealth effect.

These features are best observed in a color-coded contour plot of RCS as a function of two parameters: the normalized frequency and the apex angle-see Figs. 4 and 5. Indeed, on the upper panels of Fig. 4, which correspond to the apex-on incidence $\beta=90^{\circ}$, at the prism with $\varepsilon=2.5$, the dominant feature is a "hill" of enhanced RCS stretching at $\alpha>140^{\circ}$ and gradually tending to $\alpha=180^{\circ}$ at higher frequencies.

On the lower panels, which correspond to the base-on incidence $\beta=270^{\circ}$, new features appear in the form of the periodic areas of high RCS values along the lines $\alpha=90^{\circ}$ (this is the corner reflector effect, modulated with resonances of scatterer, see Section IV-D) and $60^{\circ}$ (this is resonances of scatterer, see Section IV-B). If the permittivity gets higher (for instance, $\varepsilon=4$ as in Fig. 5), the resonances near to $\alpha=60^{\circ}$ become more pronounced and visible even at the apex-on incidence in the both polarizations.

\section{NuMERICAL RESUltS: RESONANCES}

\section{A. Visualization of Resonances of Scatterer}

Monostatic RCS is not the best tool for observation of the resonances on a dielectric scatterer because it characterizes only the far field scattered in one (backward) direction.

Better chances to detect them are provided by plotting the frequency dependences of TSCS, which is proportional to the total power taken by the scattered field to infinity in all directions. It must be noted that any dielectric body possesses both internal and external natural modes [31], or complex poles of the field as a function of frequency. The internal modes may have high Q-factors and then get visible as peaks on the scattering spectra. They should be not mixed up with the "spurious-mode resonances" which are defects of the nonMBIE and sometimes (incorrectly) called the same name [15]. External-mode resonances normally are not visible because their Q-factors are low (see [31] for details).

The plots in Fig. 6 show the frequency scans of the TSCS for the same triangular scatterers as in Fig. 3. Here, one should 


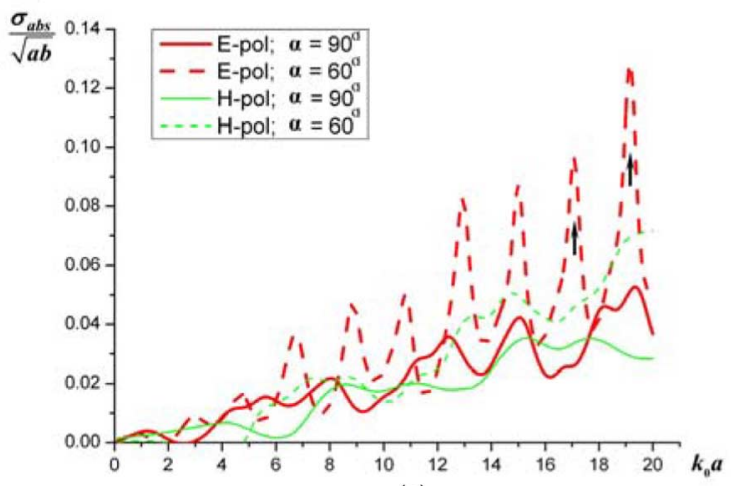

(a)

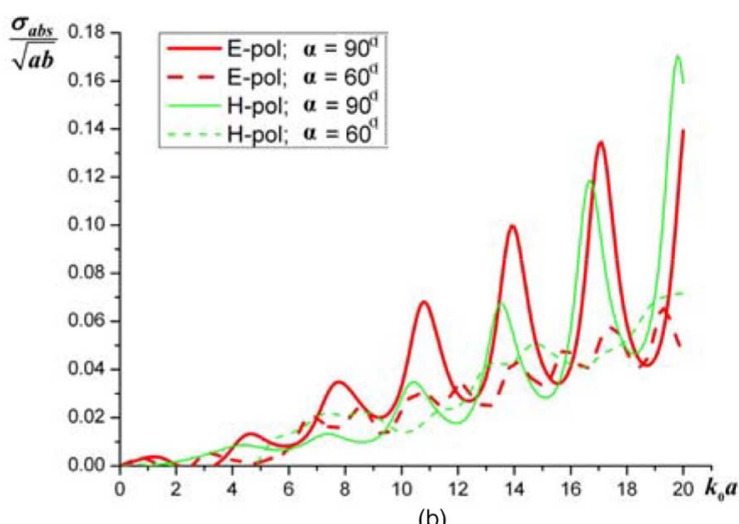

(b)

Fig. 7. Normalized ACS of a triangular dielectric cylinder with $\varepsilon=4+$ $i 10^{-2}$ as a function of the normalized frequency for two alternative polarizations at (a) $\beta=90^{\circ}$ or (b) $270^{\circ}$ and two values of the apex angle, $\alpha=60^{\circ}$ and $90^{\circ}$.

remember that, in the lossless case, the plots for $\beta=90^{\circ}$ and $270^{\circ}$ are identical due to the reciprocity theorem.

Even better tool is ACS, which is proportional to the power lost in the prism because of the bulk material losses. The positions, in frequency, of the resonances revealed by TSCS are supported by the plots of ACS shown in Fig. 7(a) and (b) computed after introducing small losses $\operatorname{Im} \varepsilon=10^{-2}$, which have almost no effect (less than 4\%) on the TSCS values.

\section{B. Resonances of Isosceles Right Triangle}

As visible from Fig. 7(b), the resonances in the right prism are best excited if it is illuminated normally to the base, in either polarization. The corresponding in-resonance field patterns (see Figs. 8 and 9) suggest that the modes can be classified as $\mathrm{E}_{44}, \mathrm{E}_{55}, \mathrm{E}_{65}$ and $\mathrm{H}_{33}, \mathrm{H}_{44}, \mathrm{H}_{55}$, respectively.

In Fig. 10, we present the contour plots of RCS of the same isosceles $90^{\circ}$ dielectric prism as a function of two parameters: the normalized frequency and the angle of incidence. The resonances discussed above overlap with the corner-reflector effect and create the area of large RCS values along the line of $\beta=270^{\circ}$ in the either of two polarizations.

The same resonances are also exited, although less efficiently, at the normal incidence on a side facet of a right triangle, i.e., at $\beta=135^{\circ}$, again in both polarizations. Note that at any $k a$ one can find a value of $\beta$, which reduces the RCS by three orders of magnitude with respect to resonances.

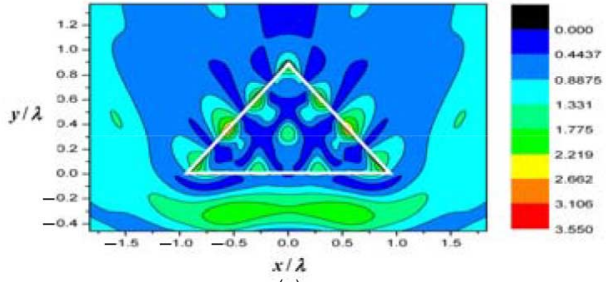

(a)

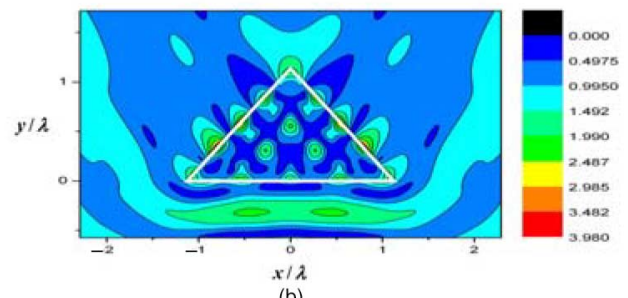

(b)

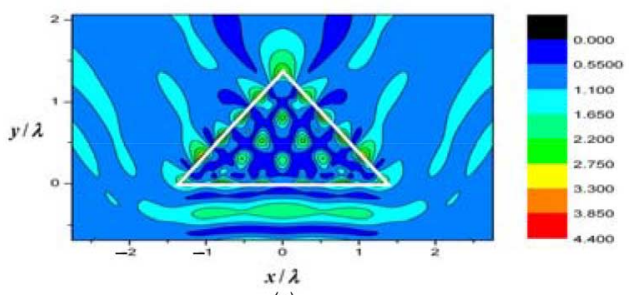

(c)

Fig. 8. Near field of a triangular cylinder with: (a) $k_{0} a=11.5$; (b) $k_{0} a=$ 14.4; and (c) $k_{0} a=17.3, \alpha=90^{\circ}, \varepsilon=4$, and $\beta=270^{\circ}$, E-wave excitation.

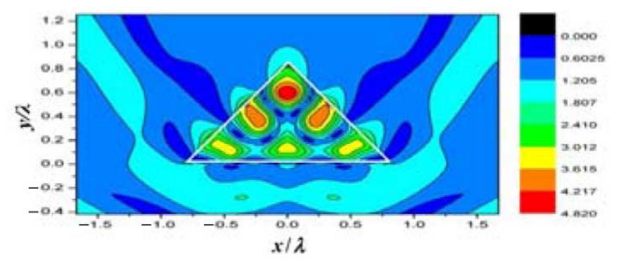

(a)

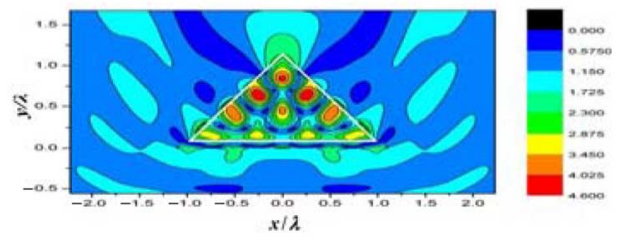

(b)

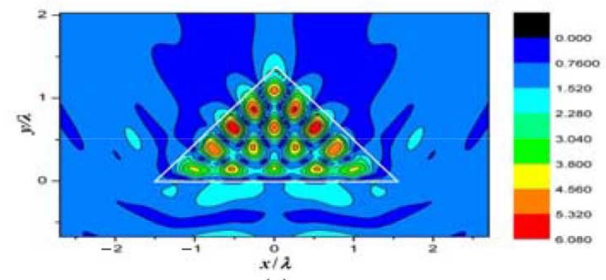

(c)

Fig. 9. Same as in Fig. 8, however, for the H-wave excitation: (a) $k_{0} a=10.5$; (b) $k_{0} a=14$; and (c) $k_{0} a=17$.

\section{Internal Resonances of Equilateral Triangle}

As already mentioned, Fig. 2(b) shows a behavior, which is drastically different from all other examples in that figure. The explanation is provided by the plots of ACS in Fig. 7(a) and (b): equilateral triangular dielectric prism supports internal resonances that are best excited in the apex-on incidence and 


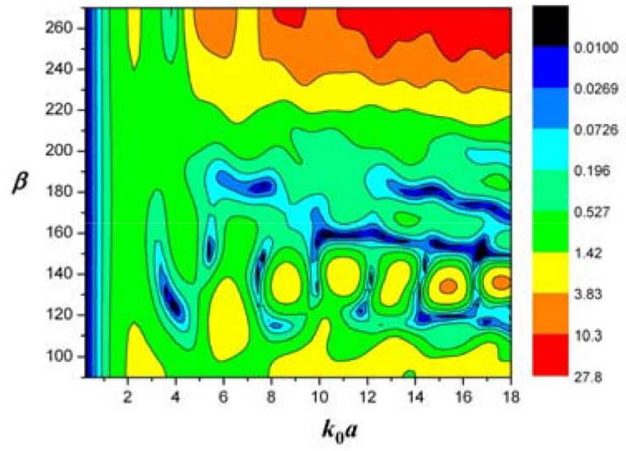

(a)

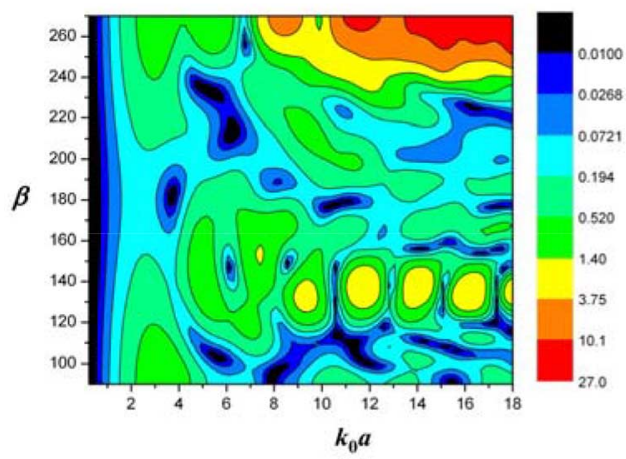

(b)

Fig. 10. Contour plots of the normalized monostatic RCS (in logarithmic scale) of a right-angle prism $\left(\alpha=90^{\circ}\right)$ with $\varepsilon=4$ as a function of the normalized frequency $k_{0} a$ and the angle of incidence $\beta$ in the (a) E-polarization case; and (b) H-polarization case.

have appreciably high Q-factors. These resonances are visible as areas of higher RCS values stretching periodically along the vertical line of $\alpha=60^{\circ}$ (Fig. 5). More accurately the corresponding resonance frequencies are found from the TSCS and ACS analysis, as explained above.

In Fig. 11, we present near fields in two such resonances in the case of the H-polarized plane wave incident on the apex. They demonstrate similar patterns with symmetric location of several field maxima inside the scatterer, their number being larger by one in (b) in comparison to (a).

At the resonance frequency, the field both in the near zone and in the far zone is dominated by the contribution of the natural-mode field, proportional to the Q-factor of the mode.

This is the reason that in Fig. 2(b) we see not only the small maxima of high reflection at the normal on-facet incidence (GO effect) but also three more intensive maxima of equal magnitudes due to the excitation of a natural mode, at the edge-on incidence.

The color-coded contour plots of RCS of the same equilateral dielectric prism are presented in Fig. 12 versus $k a$ and $\beta$. They visualize the frequencies of the internal resonances and show that they are best excited at the apex-on and facet-on incidence, with reduced RCS otherwise.

It should be noted that the effect of resonances can be found in the other cases than discussed in Sections IV-C and IV-D. For example, the appearance of the enhanced $\operatorname{RCS}$ at $\beta=30^{\circ}$ [see Fig. 2(a)] can be attributed to such effect as it takes place in only one polarization.

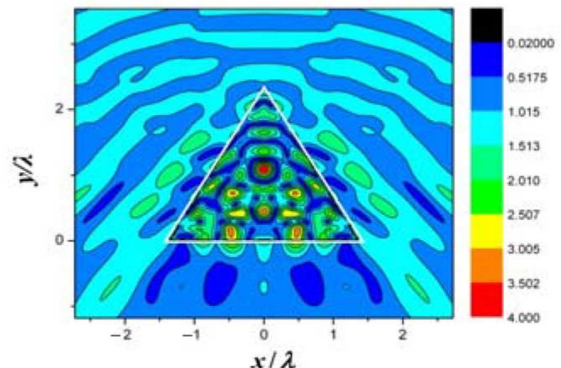

(a)

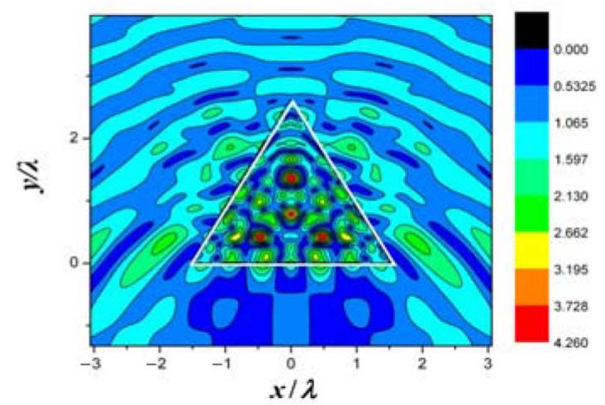

(b)

Fig. 11. Near fields of an equilateral triangular cylinder with: (a) $k_{0} a=17.1$; and (b) $k_{0} a=19.2$, and $\alpha=60^{\circ}$ and $\varepsilon=4$ under the $\mathrm{H}$-wave excitation at $\beta=90^{\circ}$ (in the maxima of ACS).

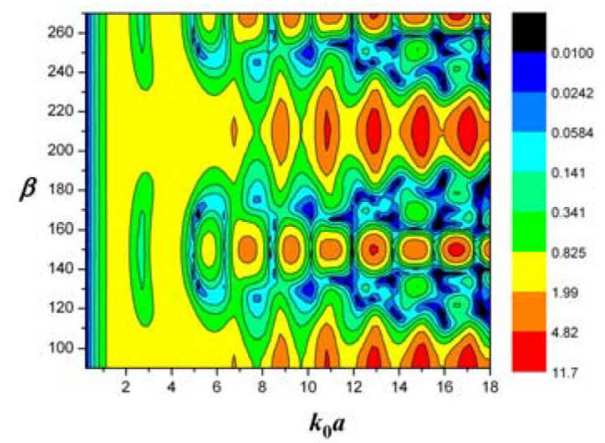

(a)

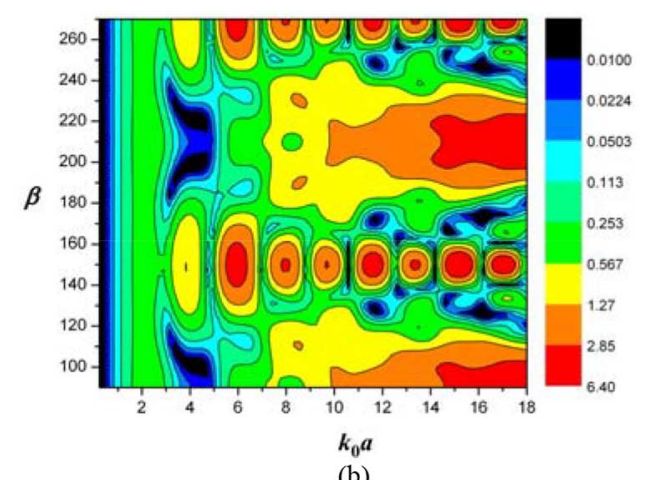

(b)

Fig. 12. Same as in Fig. 10, however, for an equilateral triangular prism ( $\alpha=$ $60^{\circ}$ ), in the (a) E-polarization case; and (b) H-polarization case.

\section{Spoiling the Resonances by Removing the Symmetry}

As shown above, symmetric configurations of dielectric prism scatterers with a right-angle isosceles shape and an equilateral-triangle shape demonstrate intensive resonances on 


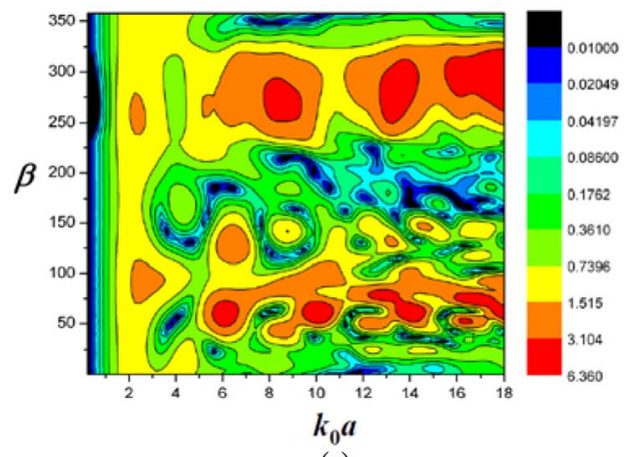

(a)

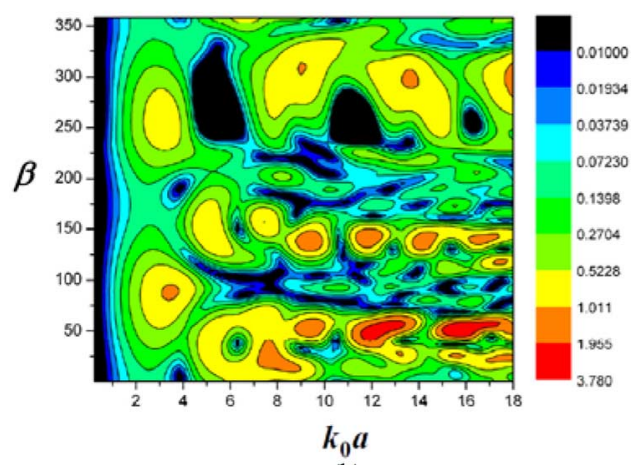

(b)

Fig. 13. Same as in Fig. 10, however, for a right-angle Pythagorean-triangle prism: (a) E-wave excitation; and (b) H-wave excitation.

the internal modes that can lead to high values of RCS. In many applications, it is desired to reduce RCS that can be done, although in narrow bands, by selecting specific orientation angle $\beta$. However a more general approach to the spoiling of resonances is related to the distortion of the shape of triangle to eliminate the symmetry. Indeed, it is known that the natural modes, which have high degree of symmetry, display higher values of $\mathrm{Q}$-factors.

Therefore in Fig. 13 we present the color-coded contour plots of $\mathrm{RCS}$, in terms of $k a$ and $\beta$, for a Pythagorean right-triangle prism [Fig. 1(b)] with the sides related as 3:4:5 and dielectric permittivity $\varepsilon=4$.

As expected, the resonances are spoiled and their effect on the RCS is 5-8 times smaller than in the case of an isosceles right-angle prism (compare maximum values of RCS to the maxima in Fig. 10). This spoiling is more pronounced in the $\mathrm{H}$-polarization case than in the E-polarization case, apparently because associated Q-factors of H-modes are usually slightly larger than those of E-modes of the same type and symmetry [31]. Similar effect takes place for the resonances in a distorted equilateral triangle (not shown).

\section{CONCLUSION}

We have presented mathematically grounded and numerically accurate analysis of the backward scattering of the E- and $\mathrm{H}$-polarized plane waves from a dielectric prism, i.e., cylinder of triangular cross-section with very small roundings. Our computational instrument has been the algorithm developed in [24] and based on the MBIE. We have studied two types of phenomena: the ray-like effects understandable from GO concepts and the resonances on the natural modes of the medium-size prism as an open resonator. Their interplay is quite complicated and both effects get more pronounced if the electric size of the scatterer becomes larger.

As we have discussed in Section III-B, the relative GO contribution grows up roughly linearly with $k a$ while the contribution of resonances depends on the nearness of the frequency to the natural-mode frequency and on the Q-factor of that mode. The latter values can grow up more dramatically with $k a$, for instance, as $\exp (\operatorname{Re} \sqrt{\varepsilon} k a)$ for the whispering-gallery modes in a lossless circular cylinder [32]. Still, as in a lossy case Q-factors of internal resonances are limited by the ratio $\operatorname{Re} \varepsilon / \operatorname{Im} \varepsilon$, one can expect that in real-life circumstances the dielectric prisms with electrical size $k a \gg 1 / \operatorname{Im} \varepsilon$ will show no resonances, i.e., display purely $\mathrm{GO}$ behaviour.

We have shown that a careful choice of the prism parameters and its orientation enables one to manipulate with RCS in broad range, reducing or enhancing it on the scale of three orders in magnitude. Here, the larger the dielectric constant of the scatterer, the more intensive the resonances even of a relatively small prism.

Our study has been focused on the RCS analysis that can be useful in the radar-target and stealth applications [28], [29]. On the other hand, the analysis of the natural modes is also important for applications in various antennas, filters, sensors, and lasers [9]-[12], [22]-[27]. This can be done using the same algorithm as we have used here, however, dealing with the roots of determinantal equations.

\section{REFERENCES}

[1] E. Hesse and Z. Ulanowski, "Scattering from long prisms computed using ray tracing combined with diffraction on facets," J. Quant. Spectrosc. Radiat. Transfer, vol. 79-80, pp. 721-732, 2003.

[2] Y.-Z. Huang, Q. Chen, W.-H. Guo, Q.-Y. Lu, and L.-J. Yu, "Mode characteristics for equilateral triangle optical resonators," IEEE J. Sel. Topics Quant. Electron., vol. 12, no. 1, pp. 59-65, Jan./Feb. 2006.

[3] C. Lafargue et al., "Localized lasing modes of triangular organic microlasers," Phys. Rev. E, vol. 90, p. 052922, 2015.

[4] J. H. Richmond, "Scattering by a dielectric cylinder of arbitrary crosssection shape," IEEE Trans. Antennas Propag., vol. 13, no. 3, pp. 334341, May 1965.

[5] J. H. Richmond, "Scattering by a dielectric cylinder of arbitrary crosssection shape," IEEE Trans. Antennas Propag., vol. 14, no. 4, pp. 460464, Jul. 1966.

[6] S.-K. Chang and K. K. Mei, "Application of the unimoment method to electromagnetic scattering of dielectric cylinders," IEEE Trans. Antennas Propag., vol. 24, no. 1, pp. 35-42, Jan. 1976.

[7] N. Morita, "Surface integral representations for electromagnetic scattering from dielectric cylinders," IEEE Trans. Antennas Propag., vol. 26, no. 2, pp. 261-266, Mar. 1978.

[8] K. Yashiro and S. Ohkawa, "Boundary element method for electromagnetic scattering from cylinders," IEEE Trans. Antennas Propag., vol. 33, no. 4, pp. 383-389, Apr. 1985.

[9] H. C. Yin and W. X. Zhang, "EM scattering from a lossy dielectric triangular cylinder based on novel coupled boundary integral equations," in Proc. IEEE Antennas Propag. Soc. Int. Symp., 1991, pp. 566-569.

[10] J. Wiersig, "Hexagonal dielectric resonators and microcrystal lasers," Phys. Rev. A, vol. 67, p. 023807, 2003.

[11] S. V. Boriskina, T. M. Benson, P. Sewell, and A. I. Nosich, "Tuning of the 2D elliptic whispering-gallery-mode microdisk waveguide filters," IEEE/OSA J. Lightwave Technol., vol. 21, no. 9, pp. 1987-1995, Sep. 2003

[12] V. Giannini and J. A. Sànchez-Gil, "Calculations of light scattering from isolated and interacting metallic nanowires of arbitrary cross section by means of Green's theorem surface integral equations in parametric form," J. Opt. Soc. Am. A, vol. 24, pp. 2822-2830, 2007. 
[13] T. Søndergaard, "Modeling of plasmonic nanostructures: Green's function integral equation methods," Phys. Stat. Sol. (b), vol. 244, pp. 34483462, 2007.

[14] M. Lucido, G. Panariello, and F. Schettino, "Scattering by arbitrary polygonal cross-section dielectric cylinders at oblique incidence," IEEE Trans. Antennas Propag., vol. 58, no. 2, pp. 540-550, Feb. 2010.

[15] A. F. Peterson, "The 'interior resonance' problem associated with surface integral equations of electromagnetics: numerical consequences and a survey of remedies," Electromagnetics, vol. 10, pp. 293-312, 1990.

[16] S. Amini and S. M. Kirkup, "Solutions of Helmholtz equation in the exterior domain by elementary boundary integral methods," J. Comput. Phys., vol. 118, pp. 208-221, 1995.

[17] E. I. Smotrova et al., "Spectra, thresholds and modal fields of a kiteshaped microcavity laser," J. Opt. Soc. Am. B, vol. 40, no. 6, pp. 1732 1742, 2013.

[18] J. L. Tsalamengas, "Exponentially converging Nystrom method in oblique diffraction of arbitrarily polarized waves by bianisotropic/chiral cylinders with arbitrary smooth cross section," IEEE Trans. Antennas Propag., vol. 61, no. 7, pp. 3362-3673, Jul. 2013.

[19] C. Muller, Foundations of the Mathematical Theory of Electromagnetic Waves. Berlin, Germany: Springer, 1969 (German edition, 1957).

[20] V. V. Solodukhov and E. N. Vasilev, "Diffraction of a plane electromagnetic wave by a dielectric cylinder of arbitrary cross section," Sov. Phys. Tech. Phys., vol. 15, no. 7, pp. 32-36, 1970.

[21] V. Rokhlin, "Rapid solution of integral equations of scattering theory in 2-D," J. Comput. Phys., vol. 86, pp. 414-439, 1990.

[22] S. V. Boriskina, P. Sewell, T. M. Benson, and A. I. Nosich, "Accurate simulation of 2D optical microcavities with uniquely solvable boundary integral equations and trigonometric Galerkin discretization," J. Opt. Soc. Am. A, vol. 21, no. 3, pp. 393-402, 2004.

[23] S. V. Boriskina, T. M. Benson, P. Sewell, and A. I. Nosich, "Optical modes in imperfect square and triangular microcavities," IEEE J. Quant. Electron., vol. 41, no. 6, pp. 857-864, Jun. 2005.

[24] I. O. Sukharevsky, O. V. Shapoval, A. I. Nosich, and A. Altintas, "Validity and limitations of the median-line integral equation technique in the scattering by material strips of sub-wavelength thickness," IEEE Trans. Antennas Propag., vol. 62, no. 7, pp. 3623-3631, Jul. 2014.

[25] G. L. Hower, R. G. Olsen, J. D. Earls, and J. B. Schneider, "Inaccuracies in numerical calculations of scattering near natural frequencies of penetrable objects," IEEE Trans Antennas Propag., vol. 41, no. 7, pp. 982 986, Jul. 1993.

[26] A. V. Boriskin et al., "Assessment of FDTD accuracy in the compact hemielliptic dielectric lens antenna analysis," IEEE Trans. Antennas Propag., vol. 56, no. 3, pp. 758-764, Mar. 2008.

[27] J. Niegemann, W. Pernice, and K. Busch, "Simulation of optical resonators using DGTD and FDTD," J. Opt. A, Pure Appl. Opt., vol. 11, no. 11 , p. $114015 / 10,2009$.

[28] G. T. Ruck, Ed., Radar Cross-Section Handbook. New York, NY, USA: Plenum-Press, 1970.

[29] C. Cugiani, R. Orta, P. Savi, and R. Tascone, "Full-wave investigation of a corner reflector proposed as reference line target for automotive applications," IEEE Trans. Antennas Propag., vol. 45, no. 12, pp. 1823-1829, Dec. 1997.

[30] O. V. Shapoval, R. Sauleau, and A. I. Nosich, "Scattering and absorption of waves by flat material strips analyzed using generalized boundary conditions and Nystrom-type algorithm," IEEE Trans. Antennas Propag., vol. 59, no. 9, pp. 3339-3346, Sep. 2011.

[31] C. P. Dettmann, G. V. Morozov, M. Siebe, and H. Waalkens, "Internal and external resonances of dielectric disks," Europ. Phys. Lett., vol. 87, pp. 34003-34006, 2009.

[32] E. I. Smotrova, A. I. Nosich, T. M. Benson, and P. Sewell, "Cold-cavity thresholds of microdisks with uniform and non-uniform gain: Quasi3D modeling with accurate 2D analysis," IEEE J. Sel. Topics Quant. Electron., vol. 11, no. 5, pp. 1135-1142, 2005.

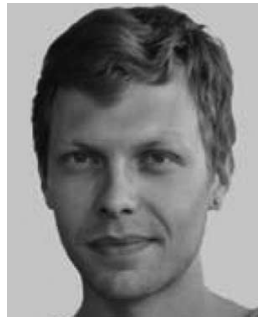

Ilya O. Sukharevsky (S'08-M'13) was born in 1984 in Kharkiv, Ukraine. He received the B.Sc. and M.Sc. degrees in mathematics from the Kharkiv National University, Kharkiv, Ukraine, and the Ph.D. degree from the Institute for Radio-Physics and Electronics of the National Academy of Sciences of Ukraine, Kharkiv, Ukraine, in 2005, 2006, and 2011, respectively.

$\mathrm{He}$ is currently a Visiting Scientist at the Communication and Spectrum Management Research Center (ISYAM), Bilkent University, Ankara, Turkey. His research interests include the integral equation techniques and asymptotic methods of electromagnetics.

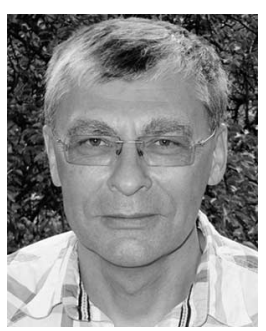

Alexander I. Nosich (M'94-SM'95-F'04) was born in Kharkiv, Ukraine, in 1953. He received the M.S., Ph.D., and D.Sc. degrees in radio physics from the Kharkiv National University, Kharkiv, Ukraine, in 1975, 1979, and 1990, respectively.

Since 1979, he has been with the Institute of Radio Physics and Electronics of the National Academy of Science of Ukraine, Kharkov, Ukraine, where he is currently a Professor and Principal Scientist. Since 2010, he also heads (initiated by him) the Laboratory of Micro and Nano Optics at this institute. Since 1992, he has held a number of Guest Fellowships and Professorships in the EU, Japan, Singapore, and Turkey. His research interests include computational electromagnetics, wave scattering, and simulation of antennas, open waveguides, and lasers.

Prof. Nosich was one of the initiators of the international conference series on Mathematical Methods in Electromagnetic Theory (MMET) held in Ukraine in 1990-2014. In 1995, he organized the IEEE East Ukraine Joint Chapter, the first in the former USSR. From 2001 to 2003, he represented Ukraine in the European Microwave Association. Since 2008, he represents Ukraine in the European Association on Antennas and Propagation.

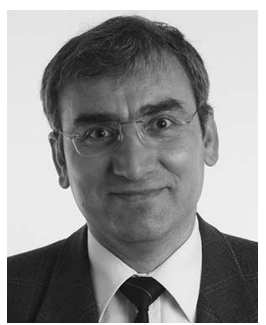

Ayhan Altintas (SM'93) received the B.S. and M.S degrees from the Middle East Technical University, Ankara, Turkey, and the Ph.D. degree from the Ohio State University (OSU), Columbus, OH, USA, in 1979, 1981, and 1986, respectively, all in electrical engineering.

$\mathrm{He}$ is currently a Professor of electrical engineering and Director of the Communication and Spectrum Management Research Center at Bilkent University, Ankara, Turkey, and Dean of the Faculty of Engineering and Natural Sciences at Abdullah Gul University, Kayseri, Turkey. His research interests include electromagnetics, antennas, propagation, and wireless communication systems.

Prof. Altintas was the Chairman of the IEEE Turkey Section in 1991-1993 and 1995-1997. He is the Founder of the IEEE AP/MTT Chapter in Turkey. At present, he is the National Chair of URSI Commission B. He was a Fullbright Scholar and an A. von Humboldt Fellow. He received the OSU ElectroScience Laboratory Outstanding Dissertation Award (1986), Research Award of Prof. M. N. Parlar Foundation (1991), Young Scientist Award of the Scientific and Technical Research Council of Turkey (1996), and the IEEE Third Millennium Medal. 